\title{
Approaches to Designing a Wireless Sensor Network Node
}

\author{
Ivan Buhrym \\ Department of Design and Operation of \\ Electronic Devices \\ Kharkiv National University of Radio \\ Electronics \\ Kharkiv, Ukraine \\ ivan.buhrym@nure.ua
}

\author{
Oleksandr Vynokurov \\ Department of Design and Operation of \\ Electronic Devices \\ Kharkiv National University of Radio \\ Electronics \\ Kharkiv, Ukraine \\ oleksandr.vynokurov2@nure.ua
}

\author{
Pavlo Galkin \\ Department of Design and Operation of \\ Electronic Devices \\ Kharkiv National University of Radio \\ Electronics \\ Kharkiv, Ukraine \\ pavlo.halkin@nure.ua
}

\begin{abstract}
The object of the research is the hardware component for building a test platform for wireless sensor networks. The aim of the work is to develop a software and hardware test platform for wireless sensor networks. As a result of the analysis, the node structures, wireless sensor network modules, CC2530 peripherals were analyzed. A module based on the CC2530 PA was chosen as the hardware. Given to optimize the structure of the node for as one of approach to designing a wireless sensor network node. Also given report about difference in approach to designing nodes and uses areas.
\end{abstract}

Keywords-approach, CC2530, software and hardware test platform, node, wireless sensor network

\section{INTRODUCTION}

This Wireless sensor networks (WSN) systems have a lot of problems like security, energy consumption, heterogeneity and other disadvantages that need be solved [1]. Therefore, it is quite difficult to design a sensor network node so that it satisfies the necessary criteria for optimality. If such a node is also used for testing and training, then additional requirements for the construction will be propose to, for example, as in articles of designing microprocessor systems [2] or embedded control systems [3]. Energy monitoring [4] is a key factor for the successful prolongation of life times each nodes in wireless sensor network, for examples reducing the power consumption of nodes [5]. Therefore, can set the task to optimize the structure of the node for as one of approach to designing a wireless sensor network node.

In conventional two-tiered WSN, sensors in each cluster transmit observed data to a fusion center via an intermediate supernode. This structure is vulnerable to supernode failure. A double supernode system model with a new coding scheme is proposed to monitor a binary data source [6]. One of design method of WSN node and gateway node for monitoring of data presented in article [7]. Each of the sensing nodes was composed of an ATmega128L microprocessor and a CC2420 transceiver module. Micro controller S3C2410X was used as the kernel of hardware platform in the gateway node [7].

Next good example of design node of WSN for aim at the problems existing in the information monitoring of the farmland environment such as the limited energy, low system stability and large monitoring area, a WSN node for rice field based on hybrid antenna is designed to realize the real-time on-line monitoring for the environmental parameters of rice fields in the network. As for the hardware, the node uses a STM32F103VET6 as a processing core, and a WLK01L39 RF chip is used in wireless communication module, while the sensor module is composed of the air temperature and humidity sensor, light intensity sensor and soil moisture sensor. As for the software, uC/OS-II is applied as an operational system to realize multitask scheduling running. The sensor node applies a mechanism as sleeping and waking up work modes to reduce power consumption. The current consumption of sensor nodes is $0.024 \mathrm{~mA}$ under the sleeping mode, $32.32 \mathrm{~mA}$ under the data collection, $26.25 \mathrm{~mA}$ under data transmission and $21.95 \mathrm{~mA}$ under the operating mode. The results of a long time networking experiment indicate that the average PLR (Packet Loss Rate) of network is $0.76 \%$. In conclusion, the design of sensor node system is suitable for the real-time and stable monitoring of rice field [8]. The paper [9] introduces the basic structure of wireless sensor network node based on ARM, and it delivers a detailed analysis on the operating features and the CC2480 hardware interface of the ZigBee processor, what's more, it specifically talks about the implementation of the Linux driver of WSN nodes. The paper [10] propose a microcontroller based anti poaching system employing WSN technology, which is capable of detecting theft by monitoring the vibrations produced by the cutting of trees/branches using a 3 axis MEMS accelerometer. A low power MSP430F5529 microcontroller is used along with Xbee RF modules based on IEEE 802.15.4 Zigbee standards to communicate to a central server from a remote place. The embedded system architecture and the hardware/software designs are described in detail. Vibration data collected by various tests on wood and simulated using Labview.

In order to solve the problem of inflexible location, dead angle and large measurement error of the network node of the wire monitoring system for factory toxic gas leakage, a toxic gas monitoring and alarm system based on CC2530 wireless sensor network is designed [11]. Therefore, after analyzing the developed and designed nodes, can define several research tasks:

- optimize the structure of the node;

- $\quad$ selection of chip for WSN node;

- peripheral requirements development. 


\section{OPTIMIZATION OF STRUCTURE NODES}

At the moment, there are two basic solutions for constructing ZigBee nodes for wireless sensor networks. In the first case, the developer is encouraged to construct the hardware unit independently, using different microprocessors supporting the IEEE 802.15.4 radio interface.

The radio transceiver in IEEE 802.15.4 complies only with the functions of receiving and transmitting information over a radio channel and does not contain software that implements the ZigBee protocol stack. The software implementation of the ZigBee stack relies on an external microcontroller to which the transceiver chip connects to one of the standard interfaces, such as a serial peripheral interface (SPI) or a parallel interface. Additionally, the transceiver requires a bunch, which includes an antenna, a quartz resonator, as well as a small number of passive components. The use of such SoC (Systems on the crystal) or SiP (Integrated hybrid chips) performs all the functions for working with ZigBee as WSN node and a microcontroller to perform a user program, though it will be less energy efficient, but in turn will be able to provide greater flexibility to execute this program in terms of Input/Output, external interfaces, or have any specific functions. This option is not the most economical in terms of the cost of chips, but it does not require much effort in design development. In the case of using a platform in a single body (PiP), the design task in general consists only in the organization of the binding of this chip. The digamma of structure chip is shown on Fig. 1

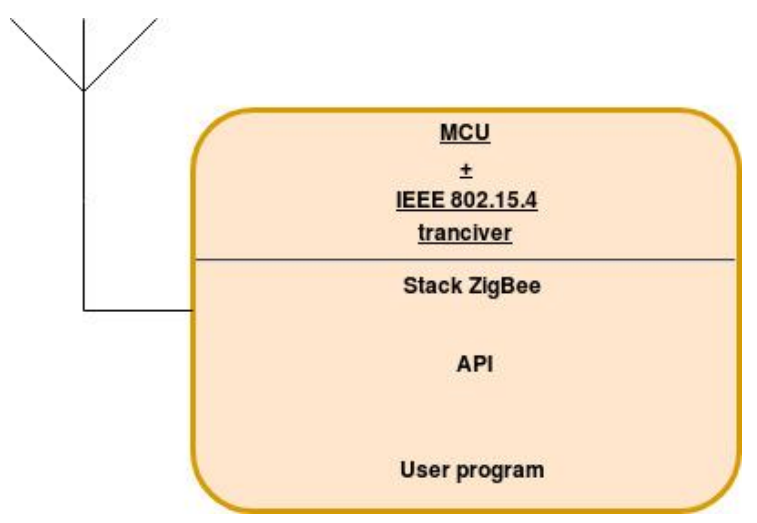

Fig. 1. Digamma of structure WSN node in one SoC

Another version with separate microcontroller with user program and radio transceiver. In this case the microcontroller has enough resources not only for realization the main functions of the program, but also the ZigBee stack. In this case, the chip The IEEE 802.15.4 radio transceiver interfaces with the base processor per means SPI interface. This structure is shown on Fig. 2.

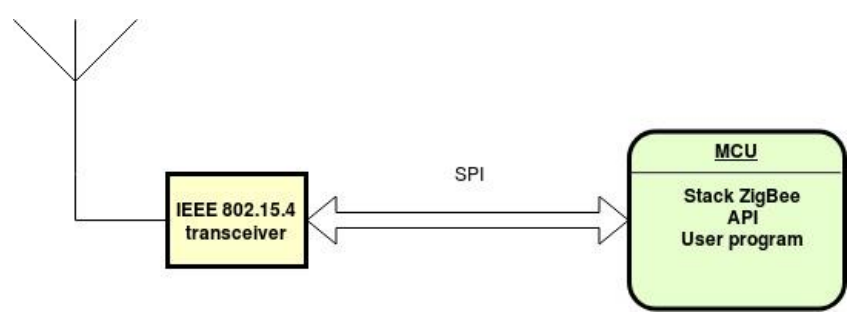

Fig. 2. Digamma of structure WSN node with separate radio transceiver and microcontroller with user program
The structure on Fig. 3 differs only in that there two microcontrollers are used: the first to implement the ZigBee stack, and the other to execute the user program, the data exchange is also carried out through the SPI. This option increases the overall power consumption.

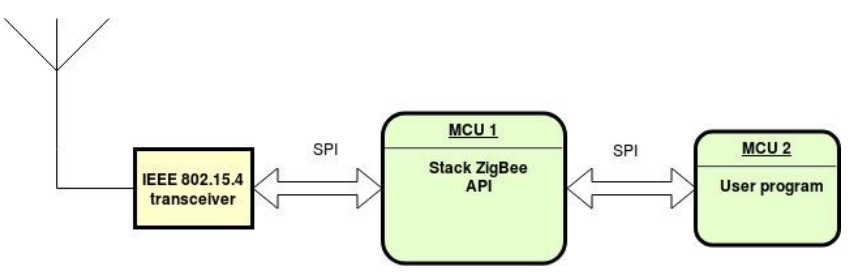

Fig. 3. Digamma of structure WSN node with two MCU and one transceiver

The closest case to the implementation of the one system on a chip is the system shown in Fig. 4, where separate microcontroller is used for user program.

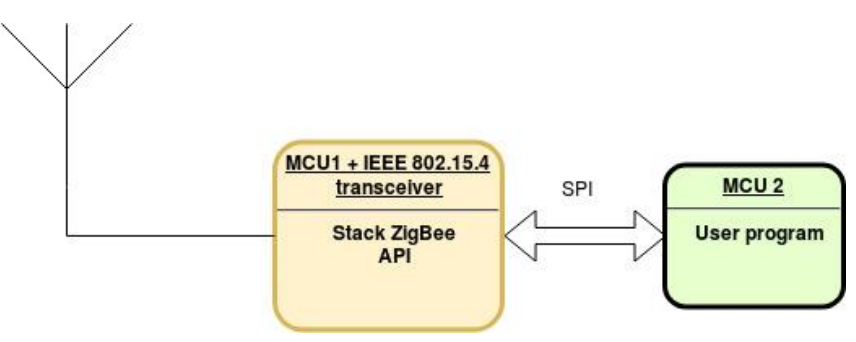

Fig. 4. Digamma of structure WSN node with one MCU for user program and one MCU with transceiver

After considering the peculiarities of the structure the node of wireless sensor networks, it should be noted difference between $\mathrm{SiP}$ and $\mathrm{SoC}$ approaches. Integrated hybrid chips, which combine in one case crystals of the "classic" radio transmitter and general purpose microcontroller. This solution allows you to place the ZigBee software stack directly in the built-in microcontroller memory and thereby significantly reduce load on an external microcontroller, which provides solution to the tasks of a particular application. These microcircuits require a minimum strap, including an antenna. Systems on the crystal - this type of chip actually replicate the capabilities of SiP, but unlike them and the transmitter receiver, and built-in the microcontroller is executed on one crystal. Some firms, such as Texas Instruments, called such crystals ZigBee processors. They interact with the external controller of the application in one of the standard interfaces (SPI, Universal Asynchronous Receiver (UART)) and allow loading in memory of network protocols of different complexity depending on the predicted the complexity and structure of the implemented network.

Therefore, we can conclude that WSN node in one SoC it is first step for optimization energy consumption of node and consequently, longer life time all network.

\section{PREPARE SELECTION OF CHIP FOR WIRELESS SENSOR NETWORK}

There are many different modules to implement ZigBee networks. Each of them has its own features, both in terms of the hardware part and in terms of software in the form of various environments for working with these modules [12]. 
There are various modules based on the SoC CC2530 [13], in essence they are a board that hosts the CC2530 itself, an external quartz resonator, several passive components, an antenna connector (or built-in antenna) and outputs for connecting to other devices. The typical node of wireless sensor network can be constructed using a chip CC2530. This transceiver can be ready for prototyping by PCB boardmodule (Fig. 5).

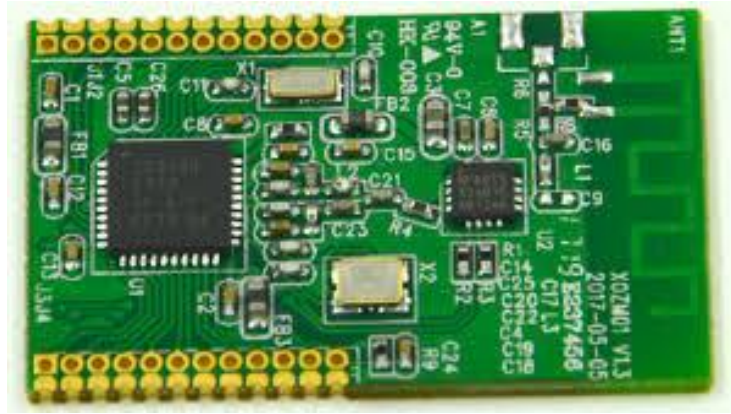

Fig. 5. PCB board-module base on chip CC2530

The use of ready-made modules is the easiest way to start working with ZigBee [14], since it does not require its own development, you only need to select the module with the required number of ports, the required data interfaces and related parameters. Such modules are often installed directly on the board with the necessary operating equipment, sensors or simply with port output. Company developers of these modules usually also make additional modules with different input / output devices, sensors and other related equipment. In addition, there are additional expansion cards (shield) designed to power the module itself, output ports on connectors, containing LEDs reflect the state of the module and additional buttons.

As an example of a debugging board for the ZigBee module can consider the CC2530 PCB base (Fig. 6) on ZigBee CC2530 Sensor (Fig. 7) from the company Texas Instruments which is designed for the modules CC2530EM.

Problems in the design of embedded systems were also considered earlier [15-17].

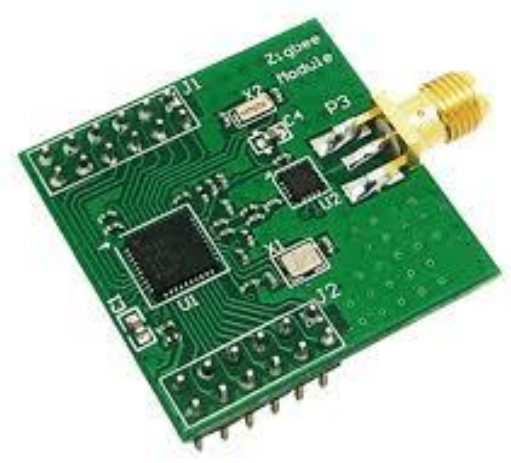

Fig. 6. Module base on chip CC2530

The ZigBee CC2530 Sensor debugging board contains:

- Full USB 2.0 interface;
- UART interface;

- LEDs and buttons;

- serial flash memory.

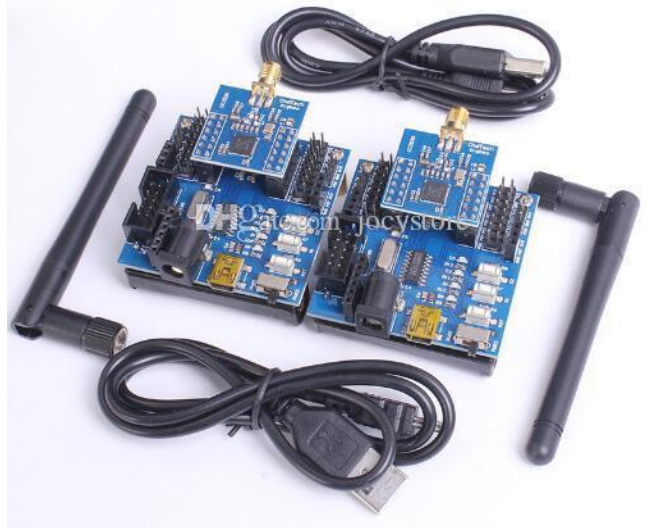

Fig. 7. ZigBee CC2530 Sensor

The CC2530 is a true system-on chip (SoC) solution for IEEE802.15.4, Zigbee and RF4CE applications [13].

\section{USING THE TEMPLATE PERIPHERAL REQUIREMENTS DEVELOPMENT}

The CC2530 contains many peripherals that provide everything to develop various applications [14]. The debugging interface uses I / O ports P21 (data) and P22 (synchronization) in debug mode. In this case, in the debug mode, the other 19 ports pins can be active, which gives a great opportunity to debug the module, while at the same time it can be connected to quite a few different devices. In other cases, the ports for debugging can work in the normal GPIO mode. In general, the debugging interface allows you to track all processes that occur in the module when it is in an active state (user program execution), and also to change the parameters of these processes (for example, values in registers) and in real time to observe changes in the work of the module.

The wiring diagram of the CC2530 is shown in Fig. 8. As can be seen in the figure, the CC2530 chip requires a very small number of external components, which greatly simplifies the development of a new product. The microcircuit is suitable for use in a variety of applications. For this, it is integrated as standard microcontroller peripherals: 8/16 bit timers; watchdog; ADC two USART modules with support for SPI and UART protocols; I / O ports (21 lines: $19 \times 4+2 \times 20 \mathrm{~mA})$ and specialized: MAC timer (defined by the IEEE 802.15.4 standard), hardware support unit for the network protocol CSMA / CA, as well as random number generator and data encryption / decryption accelerator AES algorithm using a 128-bit key. 


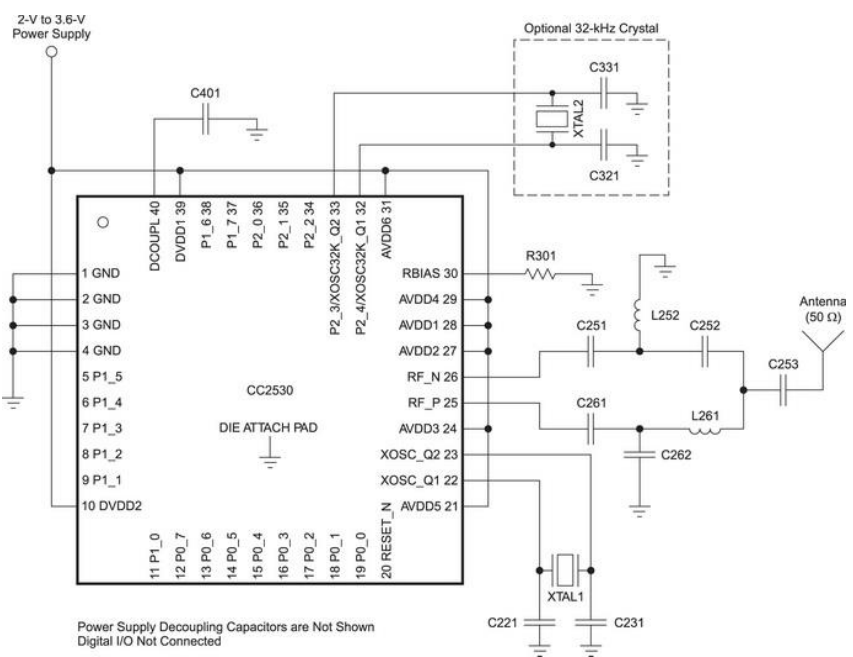

Fig. 8. Wiring diagram of the CC2530

\section{CONCLUSION}

The during of research, it was considered optimize the structure of the node, selection of chip for wireless sensor network node and discussion about peripheral requirements development. A module based on the CC2530 PA was chosen as the hardware. In article was noted that optimize of structure the wireless sensor network node as one of approach to designing a wireless sensor network with long life cycle.

\section{REFERENCES}

[1] C. Alvarado, F. Bosquez, Palacios and L. Córdoba, "Low-energy Adaptive Clustering Hierarchy protocol and optimal number of cluster head algorithm in a randomized wireless sensor network deployment," 2017 International Conference on Electrical, Electronics, Communication, Computer, and Optimization Techniques (ICEECCOT), Mysuru, 2017, pp. 1-4. oi: 10.1109/ICEECCOT.2017.8284632.

[2] V. Semenets, "Technical aspects for development laboratory base for learning FPGA and microcontroller systems," 2009 10th International Conference - The Experience of Designing and Application of CAD Systems in Microelectronics, Lviv-Polyana, 2009, pp. 145-145.

[3] P. Galkin, "Razrabotka laboratornogo kompleksa po izucheniyu vstraivaemyih sistem upravleniya i promyishlennoy avtomatizatsii [Development of a laboratory complex for the study of embedded control systems and industrial automation]," Materials of the $21 \mathrm{st}$ International Youth Forum "Radio Electronics and Youth in the 21st Century", April 25-27, 2017 Conference "Automated systems and computerized technologies of radio-electronic instrument-making", Kharkiv, KNURE, vol. 2, P.94-95. (In Russian).

[4] P. Galkin. "Analiz energopotrebleniya uzlov besprovodnih sensornih setei [Analysis of power consumption of nodes of wireless sensor networks],“ScienceRise, no.2 pp 55-61, 2014. (In Russian).
[5] P. Galkin, "Model of Reducing the Power Consumption for Node of Wireless Sensor Network in Embedded Control Systems," 2018 International Scientific-Practical Conference Problems of Infocommunications. Science and Technology (PIC S\&T), Kharkiv, Ukraine, 2018, pp. 252-256. doi: 10.1109/INFOCOMMST.2018.8631891.

[6] A. Razi, F. Afghah and A. Abedi, "Power Optimized DSTBC Assisted DMF Relaying in Wireless Sensor Networks with Redundant Super Nodes," in IEEE Transactions on Wireless Communications, vol. 12, no. 2, pp. 636-645, February 2013. doi: 10.1109/TWC.2012.121712.111968.

[7] Daozong Sun, Weixing Wang, Jianqing Lu and Zuanhui Lin, "Design of WSN nodes and network performance analysis in a tea plantation," IET International Conference on Wireless Sensor Network 2010 (IET-WSN 2010), Beijing, 2010, pp. 144-147. doi: 10.1049/cp.2010.1043.

[8] H. Chen, W. Wang, B. Sun, J. Weng and F. Tie, "Design of a WSN Node for Rice Field Based on Hybrid Antenna," 2017 International Conference on Computer Network, Electronic and Automation (ICCNEA), Xi'an, 2017, pp. 276-280. doi: 10.1109/ICCNEA.2017.101.

[9] J. Jinwu, C. Lecai and L. Renjia, "The Design of Linux Driver of WSN Nodes Based on ARM," 2011 Fourth International Conference on Intelligent Computation Technology and Automation, Shenzhen, Guangdong, 2011, pp. 761-764. doi: 10.1109/ICICTA.2011.477.

[10] S. Gaikwad, R. Patil, A. Khandare and A. Rai, "Design WSN node for protection of forest trees against poaching based on ZigBee," 2015 IEEE International Conference on Electronics, Computing and Communication Technologies (CONECCT), Bangalore, 2015, pp. 14. doi: 10.1109/CONECCT.2015.7383911.

[11] C. Ma, N. Li, X. Pang, S. Wang and Y. Yang, "Hardware Design of Factory Toxic Gas Leakage Monitoring System Based on CC2530," 2018 10th International Conference on Intelligent Human-Machine Systems and Cybernetics (IHMSC), Hangzhou, 2018, pp. 243-245. doi: 10.1109/IHMSC.2018.10162.

[12] M. Khanafer, M. Guennoun and H. T. Mouftah, "A Survey of Beacon-Enabled IEEE 802.15.4 MAC Protocols in Wireless Sensor Networks," in IEEE Communications Surveys \& Tutorials, vol. 16, no. 2, pp. 856-876, Second Quarter 2014. doi: 10.1109/SURV.2013.112613.00094.

[13] Second Generation System-on-Chip Solution for $2.4 \mathrm{GHz}$ IEEE 802.15.4 / RF4CE / ZigBee [Electronic resource] / Texas Instruments.- Available at: www/ URL: http://www.ti.com/lit/ds/symlink/cc2530.pdf.

[14] F. Knutti, N. Tobler and H. Mathis, "Low-power voting device for use in education and polls employing TI's CC2530 RF CHIP," 2014 6th European Embedded Design in Education and Research Conference (EDERC), Milano, 2014, pp. 221-224. doi: 10.1109/EDERC.2014.6924392.

[15] P. Galkin, L. Golovkina and I. Klyuchnyk, "Analysis of Single-Board Computers for IoT and IIoT Solutions in Embedded Control Systems," 2018 International Scientific-Practical Conference Problems of Infocommunications. Science and Technology (PIC S\&T), Kharkiv, Ukraine, 2018, pp. 297-302. doi: 10.1109/INFOCOMMST.2018.8632069.

[16] T. Nimi and P. Samundiswary, "Comparative analysis of ZigBee network with tree and mesh topology for different range of frequencies," 2017 2nd International Conference on Communication and Electronics Systems (ICCES), Coimbatore, 2017, pp. 560-564. doi: 10.1109/CESYS.2017.8321140.

[17] P. Galkin, "Analysis models of collection data in wireless sensor networks," 2016 Third International Scientific-Practical Conference Problems of Infocommunications Science and Technology (PIC S\&T), Kharkiv, 2016, pp. 233-236. doi: 10.1109/INFOCOMMST.2016.7905392. 